\title{
BIOCHEMICAL AND HEMATOLOGICAL REFERENCE VALUES IN CALVES AND THEIR SIGNIFICANCE FOR HEALTH CONTROL
}

\author{
J. BOUDA and P. JAGOŠ \\ Department of Diagnosis, Therapy and Prevention of Animal Diseases, \\ University of Veterinary Science, 61242 Brno
}

Received December 1, 1983

\begin{abstract}
Bouda J., P. Jagoš: Biochemical and Hematological Reference Values in Calves and Their Significance for Health Control. Acta vet. Brno, 53, 1984: 137-142.

Clinical and biochemical examination of 114 calves from birth to the age of 6 months was carried out. Blood samples were taken from v. jugularis prior to the first colostrum intake and after 1, 4, 7, 21, 42, 60, 90 and 180 days. From the total number of 114 followed calves reference biochemical and hematological values were obtained from the group of 53 healthy calves.

In dependence on calf age reference values of total protein including fractions and immunoglobulins in blood serum and levels of vitamin A, E and C, carotenes, glucose, urea, enzyme activities (AST, GMT), minerals ( $\mathrm{Na}, \mathrm{K}, \mathrm{Mg}$, inorg. $\mathrm{P}, \mathrm{Zn}, \mathrm{Cu}, \mathrm{Fe}$ ) in blood plasma were determined. In calf blood acid-base values $\left(\mathrm{pH}, \mathrm{pCO}_{2}, \mathrm{BE}\right.$, SB), hematocrit, hemoglobin, methemoglobin, erythrocyte and leukocyte counts were determined. Hypogammaglobulinaemia, hypovitaminoses $\mathrm{A}$ and $\mathrm{E}$ were found in $50-75 \%$ of calves. Among the most significant biochemical data from the diagnostic point of view, the determination of Ig and vitamin levels, acid-base balance of blood, plasma levels of glucose, urea, some minerals and AST activity are included.

The selection of clinico-biochemical parameters of calves has to be purposeful. During the examination and diagnosis of metabolic disorders the development of immunological proceses, the lability of homeostasis, the functional and organ immaturity and the dynamics in relation to calf age have to be taken into consideration.
\end{abstract}

Calf, blood, protein, Ig, vitamins, carotene, glucose, urea, enzymes, minerals, acid-base values, PCV, hemoglobin, erythrocyte and leukocyte count.

The significance of research concerning the metabolic disorders for the development of large-scale animal production lies in several factors: the disorders are the cause of losses and influence further development of an animal; moreover, they play an important role in the etiopathogenesis of factorial and infectious diseases (Bouda 1979; Jagoš et al. 1982). The control of the development of health condition in calves and especially that of metabolic disorders by clinical methods of examination is very difficult, often even impossible, since these disorders usually are of subclinical character. For this reason we have, similarly as in the case of adult animals, focused our attention to the selection of clinico-biochemical data for the evaluation of dynamics of metabolic development and to an early diagnosis of calf diseases.

When processing information from our and ample foreign literature we have found that the determination of biochemical and hematological values in calves was usually carried out only once (Vagher et al. 1973; Kitchenham et al. 1978; Slanina, Beseda 1979; Lumsden et al. 1980) or that the dynamics was studied only in a short time interval (McMurray et al. 1978).

The aim of the presented work was to determine the basic biochemical and hematological values for calves in relation to their age and in this way to elaborate and complete the system of preventive diagnosis in cattle by effective methods of calf health control. 


\section{Materials and Methods}

Clinical and laboratory examination was conducted on 114 crossbred calves (Bohemian Pied $\times$ $\times$ Black Pied Lowland) from birth to the age of six months. Blood samples were taken from v. jugularis prior to colostrum intake and then after $1,4,7,21,42,60,90$ and 180 days past the colostrum intake. Altogether nine blood samples were taken from each calf. From the total number of 114 followed calves basic biochemical and hematological data were obtained from the set of 53 calves. This set consisted of calves in good health condition. Another selection criterion was the immunoglobulin level in blood serum measured in 4-day-old calves ( $\mathrm{McEw}$ an et al. 1970). Its value did not drop below 18 turbidity units.

The following clinico-biochemical and hematological parameters were determined: total protein including fractions, and immunoglobulins in the blood serum, levels of vitamin $\mathrm{A}, \mathrm{E}$ and $\mathrm{C}$, carotens, glucose, urea, enzyme activities (AST, GMT, ALP, LD), minerals ( $\mathrm{Na}, \mathrm{K}, \mathrm{Ca}, \mathrm{Mg}$, inorg. $\mathrm{P}, \mathrm{Zn}, \mathrm{Cu}, \mathrm{Fe})$ in the blood plasma. In the blood, acid-base values $\left(\mathrm{pH}, \mathrm{pCO}_{2}, \mathrm{BE}, \mathrm{SB}\right)$, hematocrit, hemoglobin, methemoglobin, erythrocyte and leukocyte count were determined. Usual methods were employed for the determination of individual biochemical and hematological values (Jagoš et al. 1980).

\section{Results and Discussion}

Reference biochemical and hematological values in blood, blood plasma and serum of calves are presented in Tables 1 to 4. The most important reference values in calf blood are summarized in Table 5.

Table 1

Reference values in calf blood

\begin{tabular}{|c|c|c|c|c|c|c|c|c|c|c|c|c|c|c|}
\hline \multirow[t]{2}{*}{$\begin{array}{l}\text { Calf } \\
\text { age }\end{array}$} & \multicolumn{2}{|c|}{$\mathrm{pH}$} & \multicolumn{2}{|c|}{$\underset{1 \mathrm{~Pa}}{\mathrm{pCO}_{2}}$} & \multicolumn{2}{|c|}{$\begin{array}{c}\text { Base excess } \\
\mathrm{mmol} / 1\end{array}$} & \multicolumn{2}{|c|}{$\underset{\mathbf{g} / 1}{\text { Hemoglobin }}$} & \multicolumn{2}{|c|}{$\underset{1 / 1}{\text { Hematocrit }}$} & \multicolumn{2}{|c|}{$\begin{array}{c}\text { Erythro- } \\
\text { cytes } \\
\mathrm{T} / 1\end{array}$} & \multicolumn{2}{|c|}{$\begin{array}{c}\text { Leukocytes } \\
\text { G/1 }\end{array}$} \\
\hline & $\bar{x}$ & 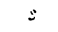 & $\bar{x}$ & $s$ & $\bar{x}$ & $s$ & $\bar{x}$ & $s$ & $\bar{x}$ & $s$ & $\bar{x}$ & $s$ & $\bar{x}$ & $s$ \\
\hline $\begin{array}{l}\text { Without } \\
\text { colostrum } \\
1 \text { day } \\
4 \text { days } \\
1 \text { week } \\
3 \text { weeks } \\
6 \text { weeks } \\
2 \text { months } \\
3 \text { months } \\
6 \text { months }\end{array}$ & $\begin{array}{l}7.264 \\
7.365 \\
7.373 \\
7.352 \\
7.366 \\
7.358 \\
7.356 \\
7.356 \\
7.358\end{array}$ & $\begin{array}{l}0.03 \\
0.04 \\
0.05 \\
0.05 \\
0.03 \\
0.03 \\
0.04 \\
0.04 \\
0.02\end{array}$ & $\begin{array}{l}8.4 \\
6.4 \\
6.3 \\
6.6 \\
6.6 \\
6.5 \\
6.5 \\
6.5 \\
6.6\end{array}$ & $\begin{array}{l}1.3 \\
1.0 \\
0.8 \\
1.3 \\
0.9 \\
0.6 \\
0.8 \\
1.1 \\
0.7\end{array}$ & $\begin{array}{l}-1.5 \\
+2.0 \\
+2.1 \\
+0.6 \\
+1.5 \\
+0.3 \\
+0.8 \\
+0.9 \\
+1.6\end{array}$ & $\begin{array}{l}2.7 \\
2.8 \\
2.7 \\
2.8 \\
2.5 \\
2.5 \\
2.4 \\
3.0 \\
3.2\end{array}$ & $\begin{array}{l}124.3 \\
113.1 \\
113.0 \\
112.7 \\
109.2 \\
103.1 \\
109.3 \\
112.3 \\
108.2\end{array}$ & $\begin{array}{l}18.4 \\
18.2 \\
19.1 \\
18.1 \\
18.2 \\
15.9 \\
13.2 \\
12.2 \\
12.7\end{array}$ & $\begin{array}{l}0.40 \\
0.34 \\
0.34 \\
0.32 \\
0.32 \\
0.32 \\
0.30 \\
0.34 \\
0.28\end{array}$ & $\begin{array}{l}0.06 \\
0.06 \\
0.05 \\
0.05 \\
0.06 \\
0.05 \\
0.03 \\
0.05 \\
0.03\end{array}$ & $\begin{array}{l}7.65 \\
6.91 \\
6.90 \\
6.68 \\
6.39 \\
6.49 \\
5.90 \\
7.08 \\
5.93\end{array}$ & $\begin{array}{l}1.37 \\
1.11 \\
1.31 \\
1.07 \\
0.09 \\
0.95 \\
0.95 \\
0.72 \\
1.39\end{array}$ & $\begin{array}{r}10.4 \\
9.8 \\
9.6 \\
9.0 \\
8.8 \\
7.9 \\
8.1 \\
9.3 \\
10.0\end{array}$ & $\begin{array}{l}3.8 \\
2.8 \\
3.3 \\
3.7 \\
2.8 \\
2.8 \\
1.8 \\
2.1 \\
2.6\end{array}$ \\
\hline
\end{tabular}

Table 2

Reference values in blood plasma of calves

\begin{tabular}{|c|c|c|c|c|c|c|c|c|c|c|c|c|c|c|c|c|}
\hline \multirow[t]{2}{*}{$\begin{array}{l}\text { Calf } \\
\text { age }\end{array}$} & \multicolumn{2}{|c|}{$\begin{array}{c}\text { Vitamin } \\
\mathrm{A} \\
\mu \mathrm{mol} / 1\end{array}$} & \multicolumn{2}{|c|}{$\underset{\mu \mathrm{mol} / 1}{\mathrm{Citamin}}$} & \multicolumn{2}{|c|}{$\underset{\mathrm{E}}{\mu \mathrm{mol} / 1}$} & \multicolumn{2}{|c|}{$\underset{\mu \mathrm{mol} / 1}{\text { Carotene }}$} & \multicolumn{2}{|c|}{$\begin{array}{c}\text { Alkaline } \\
\text { phosphatase } \\
\mu \mathrm{kat} / 1\end{array}$} & \multicolumn{2}{|c|}{$\begin{array}{c}\text { Lactate } \\
\text { dehydro- } \\
\text { genase } \\
\mu \text { kat/1 }\end{array}$} & \multicolumn{2}{|c|}{$\begin{array}{c}\text { Aspartate } \\
\text { amino } \\
\text { transferase } \\
\mu \text { kat } / 1\end{array}$} & \multicolumn{2}{|c|}{$\begin{array}{c}\text { Gama- } \\
\text {-glutamyl } \\
\text { transferase } \\
\mu \text { kat } / 1\end{array}$} \\
\hline & $\overline{\boldsymbol{x}}$ & $s$ & $\bar{x}$ & $s$ & $\bar{x}$ & $s$ & $\overline{\boldsymbol{x}}$ & $s$ & $\overline{\boldsymbol{x}}$ & $s$ & $\overline{\bar{x}}$ & $s$ & $\tilde{\tilde{x}}$ & $s$ & $\dot{\tilde{x}}$ & $s$ \\
\hline $\begin{array}{l}\text { Without } \\
\text { colostrum } \\
1 \text { day } \\
4 \text { days } \\
1 \text { week } \\
3 \text { weeks } \\
6 \text { weeks } \\
2 \text { months } \\
3 \text { months } \\
6 \text { months }\end{array}$ & $\begin{array}{l}0.29 \\
0.74 \\
0.70 \\
0.72 \\
0.79 \\
0.92 \\
1.09 \\
1.27 \\
1.72\end{array}$ & $\begin{array}{l}0.0 \\
0.3 \\
0.2 \\
0.2 \\
0.2 \\
0.1 \\
0.3 \\
0.2 \\
0.2\end{array}$ & $\begin{array}{c}83.0 \\
56.8 \\
51.2 \\
40.0 \\
41.4 \\
38.2 \\
38.0 \\
34.9 \\
-\end{array}$ & $\begin{array}{c}50.2 \\
17.3 \\
12.4 \\
17.3 \\
22.6 \\
17.7 \\
16.9 \\
6.4 \\
-\end{array}$ & $\begin{array}{l}2.1 \\
3.9 \\
4.2 \\
3.8 \\
3.9 \\
4.3 \\
5.4 \\
5.0 \\
9.5\end{array}$ & $\begin{array}{l}2.3 \\
1.8 \\
1.6 \\
1.6 \\
1.4 \\
1.2 \\
2.0 \\
1.6 \\
3.1\end{array}$ & $\begin{array}{l}0.05 \\
0.27 \\
0.26 \\
0.22 \\
0.14 \\
0.12 \\
0.63 \\
0.93 \\
4.22\end{array}$ & $\begin{array}{l}0.04 \\
0.14 \\
0.15 \\
0.15 \\
0.08 \\
0.08 \\
0.46 \\
0.53 \\
2.55\end{array}$ & $\begin{array}{l}3.1 \\
8.9 \\
3.9 \\
2.8 \\
2.3 \\
2.5 \\
2.4 \\
2.0 \\
2.3\end{array}$ & $\begin{array}{l}1.1 \\
6.9 \\
1.4 \\
1.1 \\
0.8 \\
0.9 \\
0.9 \\
0.5 \\
0.6\end{array}$ & $\begin{array}{r}8.9 \\
10.7 \\
10.2 \\
9.1 \\
11.4 \\
13.1 \\
15.4 \\
15.4 \\
15.8\end{array}$ & $\begin{array}{l}2.4 \\
2.1 \\
2.6 \\
2.2 \\
3.5 \\
2.9 \\
3.1 \\
2.0 \\
1.5\end{array}$ & $\begin{array}{l}0.22 \\
0.32 \\
0.22 \\
0.21 \\
0.26 \\
0.35 \\
0.53 \\
0.35 \\
0.33\end{array}$ & $\begin{array}{l}0.07 \\
0.09 \\
0.06 \\
0.07 \\
0.09 \\
0.14 \\
0.24 \\
0.10 \\
0.06\end{array}$ & $\begin{array}{r}0.97 \\
20.09 \\
7.36 \\
4.48 \\
1.25 \\
0.67 \\
0.68 \\
0.53 \\
0.54\end{array}$ & $\begin{array}{r}0.47 \\
18.88 \\
6.37 \\
3.81 \\
0.80 \\
0.21 \\
0.26 \\
0.11 \\
0.08\end{array}$ \\
\hline
\end{tabular}


Table 3

Reference values in blood plasma of calves

\begin{tabular}{|c|c|c|c|c|c|c|c|c|c|c|c|c|c|c|c|c|}
\hline \multirow[t]{2}{*}{$\begin{array}{l}\text { Calf } \\
\text { age }\end{array}$} & \multicolumn{2}{|c|}{$\underset{\mathrm{mmol} / 1}{\mathrm{Ca}}$} & \multicolumn{2}{|c|}{$\begin{array}{r}\text { Inorg. } P \\
\mathrm{mmol} / 1\end{array}$} & \multicolumn{2}{|c|}{$\underset{\mathrm{mmol} / \mathrm{l}}{\mathrm{Mg}}$} & \multicolumn{2}{|c|}{$\underset{\mathrm{mmol} / \mathrm{l}}{\mathrm{Na}}$} & \multicolumn{2}{|c|}{$\underset{\mathrm{mmol} / 1}{\mathrm{~K}}$} & \multicolumn{2}{|c|}{$\underset{\mu \mathrm{mol} / 1}{\mathrm{Zn}}$} & \multicolumn{2}{|c|}{$\underset{\mu \mathrm{mol} / 1}{\mathrm{Cu}}$} & \multicolumn{2}{|c|}{$\underset{\mu \mathrm{mol} / 1}{\mathrm{Fe}}$} \\
\hline & $\bar{x}$ & $s$ & $\bar{x}$ & $s$ & $\bar{x}$ & $s$ & $\bar{x}$ & $s$ & $\bar{x}$ & $s$ & $\bar{x}$ & $s$ & & s & $\bar{x}$ & $s$ \\
\hline Witl & & & & & & & & & & & & & & & & \\
\hline & 2.76 & 0.16 & 3.0 & 0.8 & 0.92 & 0.10 & 149.7 & 4 & 5.3 & 0.4 & 43.2 & 25.2 & 6.5 & & 38.3 & 12.7 \\
\hline & 2. & & & & 0.96 & & 1 & & 5.6 & 0 & & & & & & 13.5 \\
\hline & $\begin{array}{l}2.81 \\
2.74\end{array}$ & $\begin{array}{l}0 \\
0\end{array}$ & 2. & $\begin{array}{l}0 \\
0\end{array}$ & $\begin{array}{l}0.88 \\
0.83\end{array}$ & 0.11 & 14 & $\begin{array}{l}5 \\
5\end{array}$ & $\begin{array}{l}5.3 \\
5.4\end{array}$ & 0 & & & & $\begin{array}{l}3 \\
3\end{array}$ & & $\begin{array}{l}8.2 \\
9.5\end{array}$ \\
\hline & 2.76 & 0 & 2. & & 0.83 & 0. & 14 & & 5. & 0 & 38 & 14 & 17.0 & & & 13.7 \\
\hline & 2. & 0 & 2. & 0 & 0.8 & 0. & 14 & 3 & 5.3 & 0 & 3. & 10 & 15.2 & 3 & 34 & 10.2 \\
\hline & 2. & 0 & & & & & & & 5.3 & & & & 14 & & & 9.3 \\
\hline $3 \mathrm{n}$ & 2.6 & 0.2 & 2. & 0. & 0.9 & 0. & 145.1 & 3. & 5.1 & 0. & 27 & 6 & 14.0 & 2 & 36 & 9.2 \\
\hline $6 \mathrm{mo}$ & 2.5 & 0 & 3.22 & 0. & 0.98 & 0.0 & 136.6 & 5. & 4.7 & & 37.7 & 14.9 & 15.3 & 2.1 & 28.7 & 8.3 \\
\hline
\end{tabular}

Table 4

Reference values in blood serum of calves (glucose and urea in blood plasma)

\begin{tabular}{|c|c|c|c|c|c|c|c|c|c|c|c|c|c|c|c|c|}
\hline \multirow{2}{*}{$\begin{array}{l}\text { Calf } \\
\text { age }\end{array}$} & \multicolumn{2}{|c|}{$\begin{array}{l}\text { Imuno- } \\
\text { globulins } \\
\text { units }\end{array}$} & \multicolumn{2}{|c|}{$\begin{array}{c}\text { Total } \\
\text { protein } \\
\mathbf{g} / \mathbf{l}\end{array}$} & \multicolumn{2}{|c|}{$\underset{\mathrm{g} / 1}{\text { Albumins }}$} & \multicolumn{2}{|c|}{$\begin{array}{l}\text { Alpha } \\
\text { globulins } \\
\mathbf{g} / 1\end{array}$} & \multicolumn{2}{|c|}{$\underset{\mathbf{g} / \mathbf{l}}{\text { Beta }}$} & \multicolumn{2}{|c|}{$\begin{array}{c}\text { Gamma } \\
\text { globulins } \\
\mathbf{g} / \mathbf{1}\end{array}$} & \multicolumn{2}{|c|}{$\begin{array}{l}\text { Glucose } \\
\mathrm{mmol} / 1\end{array}$} & \multicolumn{2}{|c|}{$\begin{array}{c}\text { Urea } \\
\mathrm{mmol} / 1\end{array}$} \\
\hline & & & & $s$ & $\bar{x}$ & $s$ & & $s$ & & $s$ & & $s$ & $\bar{x}$ & s & $\bar{x}$ & s \\
\hline $\begin{array}{l}\text { Without } \\
\text { colostrum } \\
1 \text { day } \\
4 \text { days } \\
1 \text { week } \\
3 \text { weeks } \\
6 \text { weeks } \\
2 \text { months } \\
3 \text { months } \\
6 \text { months }\end{array}$ & $\begin{array}{l}1.0 \\
24.3 \\
22.21 \\
= \\
= \\
= \\
= \\
-\end{array}$ & $\begin{array}{l}0.9 \\
6.2 \\
6.0 \\
- \\
= \\
- \\
= \\
-\end{array}$ & $\begin{array}{l}45.3 \\
60.7 \\
59.4 \\
59.6 \\
54.8 \\
53.5 \\
58.8 \\
61.8 \\
66.7\end{array}$ & $\begin{array}{l}3.6 \\
8.3 \\
7.6 \\
7.7 \\
5.2 \\
5.2 \\
4.7 \\
6.9 \\
6.9\end{array}$ & $\begin{array}{c}26.1 \\
25.1 \\
25.2 \\
24.7 \\
30.7 \\
30.6 \\
32.1 \\
36.6 \\
-\end{array}$ & $\begin{array}{l}4.6 \\
5.8 \\
5.3 \\
4.7 \\
3.8 \\
4.7 \\
5.2 \\
5.1 \\
-\end{array}$ & $\begin{array}{r}13.0 \\
12.8 \\
11.6 \\
11.9 \\
6.4 \\
4.6 \\
5.3 \\
3.4 \\
-\end{array}$ & $\begin{array}{l}2.4 \\
3.1 \\
2.3 \\
2.3 \\
2.9 \\
1.9 \\
2.5 \\
1.5 \\
-\end{array}$ & $\begin{array}{l}5.0 \\
7.4 \\
7.4 \\
9.7 \\
8.7 \\
9.5 \\
9.1 \\
8.9 \\
-\end{array}$ & $\begin{array}{c}2.1 \\
2.9 \\
2.0 \\
3.1 \\
1.6 \\
1.9 \\
1.9 \\
2.2 \\
-\end{array}$ & $\begin{array}{r}1.1 \\
15.2 \\
14.6 \\
13.3 \\
8.9 \\
8.8 \\
12.1 \\
12.7 \\
-\end{array}$ & $\begin{array}{l}0.9 \\
5.6 \\
5.0 \\
4.5 \\
3.1 \\
3.0 \\
3.1 \\
3.3 \\
-\end{array}$ & $\begin{array}{l}4.1 \\
6.6 \\
5.4 \\
5.0 \\
5.0 \\
5.3 \\
4.4 \\
4.3 \\
4.6\end{array}$ & $\begin{array}{l}1.4 \\
2.0 \\
1.2 \\
1.1 \\
1.3 \\
0.9 \\
0.5 \\
0.6 \\
0.4\end{array}$ & $\begin{array}{l}4.6 \\
4.5 \\
5.2 \\
7.0 \\
7.2 \\
7.0 \\
6.1 \\
5.7 \\
4.4\end{array}$ & $\begin{array}{l}1.2 \\
1.6 \\
1.6 \\
1.8 \\
1.4 \\
1.5 \\
1.3 \\
1.3 \\
1.2\end{array}$ \\
\hline
\end{tabular}

Table 5

The most important reference biochemical and hematological values in calves (after colostrum intake)
1. Imunoglobulins (blood serum)
2. Total protein (blood serum after colostrum intake)
3. Vitamin A (blood plasma)
4. Vitamin E (blood plasma)
5. Gamma-globulins (blood serum)
6. $\mathrm{pH}$ value of blood
7. $\mathrm{pCO}_{2}$ of blood
8. Base excess of blood
9. Hematocrit (blood)
10. AST (GOT) (blood plasma)
11. Hemoglobin (blood)
12. Magnesium (blood plasma)
13. Calcium (blood plasma)
14. Inorg. phosphorus (blood plasma)
15. Sodium (blood plasma)
16. Iron (blood plasma)
17. Copper (blood plasma)
18. Zinc (blood plasma)
19. Urea (blood plasma)
20. Glucose (blood plasma)
21. Protein in colostral whey from the first milking 22. Methemoglobin (blood)

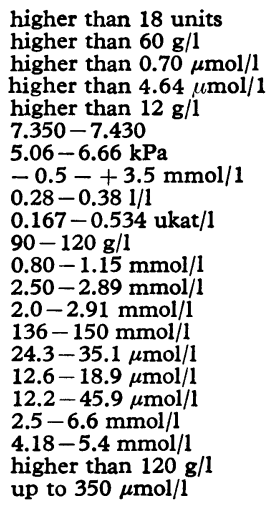

In the first developmental phase the metabolic level is determined to a high extent by metabolism of a pregnant cow, influencing the development of a young animal both in prenatal and postnatal period. When making an investigation and diagnosis of metabolic disturbances it is, however, necessary, as far as calves 
are concerned, to take into consideration the development of immunological processes, the homeostasis lability, the functional and organ immaturity and the dynamics of changes and their dependence on age (Bouda 1979; Jagoš et al. 1982). The choice of clinico-biochemical data in calves has to be purposeful, it has to start from the previous history, veterinary and production analysis and from clinical examination.

The diagnosis of hypogammaglobulinaemia, first of all in the relation of colostral immunity in calves, is based on the determination of immunoglobulins, gamma-globulins and possibly of total protein in blood serum of calves $2-5$ days old. The Ig value in this period should not decrease below 18-20 turbidity units, that of gamma globulins not below $10-12 \mathrm{~g} / \mathrm{l}$. For a rapid diagnosis of hypogammaglobulinaemia in calves in field conditions the turbidimetric determination of Ig in blood serum is satisfactory.

Hypogammaglobulinaemia, as well as hypovitaminosis A, are detected in calves very frequently, in some herds in as many as $50-75 \%$ of calves (Jagoš et al. 1980). Since the diagnosis of hypovitaminoses on the basis of clinical signs is very questionable, the determination of vitamin $\mathrm{A}, \mathrm{E}$ or $\mathrm{C}$ in blood plasma, as well as that of thiamine when cerebrocortical necrosis in calves is suspected, is justified. Prior to colostrum intake the vitamin A levels in calf blood plasma are below $0.35 \mu \mathrm{mol} / 1$ (less than $100 \mu \mathrm{g} / \mathrm{l}$ ) (Bouda et al. 1980). During the colostral nutrition of calves the plasma vitamin A level should reach above $0.7 \mu \mathrm{mol} / 1$ $(200 \mu \mathrm{g} / \mathrm{l})$. Plasma vitamin E levels in calves prior to colostrum intake are also very low, outlasting for a longer time if vitamin $\mathrm{E}$ is not supplemented (Bouda et al. 1980).

The determination of acid-base balance (Bouda 1975) is one of the important biochemical indices. In newborn, clinically healthy calves immediately after birth the $\mathrm{pH}$ value of venous blood is lower, while $\mathrm{pCO}_{2}$ is increased. Our results concerning the acid-base values are in accordance with the values found by Maurer - Schweizer and Walser (1976). Frequent shift of acid-base balance to acidity is found in the blood of calves changing from native milk to milk replacers and from milk to plant nutrition.

When evaluating hematological values of calves it is necessary to take into account physiological particularities in connection with development and growth. Hydrolability of calves, particularly a frequent occurrence of dehydration, substantially influence the hematological values. Homeostasis in calves may be disturbed by the immaturity of enzymatic system, first of all by not respecting the phase of functional maturity of digestive system in the first three weeks after birth (Bouda 1975). From the hemoglobin and hematocrit values as well as from the iron concentration in blood plasma it is possible to reveal the cases of anemia.

Increased AST activities and lowered vitamin $\mathrm{E}$ concentrations are found in calves 2-4 months old, $i$. e. in the period of increased occurrence of myodystrophies (Bouda et al. 1980).

As for minerals contained in calf blood plasma, attention should be paid first of all to the concentration of $\mathrm{Na}, \mathrm{K}, \mathrm{Ca}, \mathrm{Mg}$, inorg. $\mathrm{P}, \mathrm{Fe}, \mathrm{Cu}, \mathrm{Zn}$ and $\mathrm{J}$. The inorganic $\mathrm{P}$ concentration in calf blood plasma is significantly higher than that in cows. The changes in $\mathrm{Na}$ and $\mathrm{K}$ concentrations are detected mostly in diarrhoea, as a consequence of dehydration and acidosis. The lowest $\mathrm{Mg}$ concentrations in blood plasma are found in calves $1-4$ months old.

Increased urea levels in blood plasma are found in calves suffering from dia- 
rrhoea and are indicative of the increased dietary intake of nitrogen compounds. The lowest glucose concentrations in blood plasma occur in calves prior to colostrum intake, during plant nutrition, being however higher than reference values for dairy cows (Jagoš et al. 1975).

\section{Biochemické a hematologické referenční hodnoty u telat a jejich význam při aktivní kontrole zdraví}

Bylo provedeno klinické a laboratorní vyšetření u 114 telat od narození do stáŕí 6 měsíců. Krev u telat byla odebírána $\mathrm{z} v$. jugularis před vypitím první dávky kolostra, za 1, 4, 7, 21, 60, 90 a 180 dní. $Z$ celkového počtu sledovaných 114 telat byly referenční biochemické a hematologické hodnoty získány na souboru 53 zdravých telat.

$\mathrm{V}$ závislosti na věku telat byly určeny referenční hodnoty $\mathrm{v}$ krevním séru pro celkovou bílkovinu včetně frakcí, imunoglobuliny, $\mathrm{v}$ krevní plazmě pro vitamin A, E, C, karoteny, glukózu, močovinu, aktivity enzymů (AST, GMT), minerálie $(\mathrm{Na}, \mathrm{K}, \mathrm{Ca}$, anorg. $\mathrm{P}, \mathrm{Zn}, \mathrm{Cu}, \mathrm{Fe}$ ). $\mathrm{V}$ krvi telat byly stanoveny acidobazické hodnoty krve ( $\left.\mathrm{pH}, \mathrm{pCO}_{2}, \mathrm{BE}, \mathrm{SB}\right)$, hematokrit, hemoglobin, počet erytrocytů a leukocytů.

Hypogamaglobulinemie, hypovitaminóza A a $\mathrm{E}$ jsou zjištovány u $50-75 \%$ telat. Mezi nejvýznamnější biochemické ukazatele $\mathrm{z}$ diagnostického hlediska u telat náleži stanovení Ig, vitaminů, acidobazické rovnováhy krve, plazmatické hladiny glukózy, močoviny, některých minerálií a aktivity AST.

Výběr klinickobiochemických ukazatelů u telat musí být cílený. Při zkoumání a diagnostice poruch metabolismu $u$ telat je nutné přihlížet $k$ vývoji imunologických procesů, $\mathrm{k}$ labilitě homeostázy, $\mathrm{k}$ funkční a orgánové nezralosti a $\mathrm{k}$ dynamice $\mathrm{v}$ závislosti na věku telete.

\section{Биохимические и гематологические реферативные данные у телят и их значение при акгивном конгроле здоровья}

Проводились клинические и лабораторные исследования 114 телят со дня рождения до возраста 6 месяцев. Кровь телят брали из яремной вены перед потреблением первой дозы молозива, через $1,4,7,21,60,90$ и 180 дней. Из общего количества исследуемых 114 телят реферативные биохимичегкие и гематологические величины были получены у группы 53 здоровых телят.

В зависимости от возраста телят были определены реферативные величины в кровяной сыворотке для общего белка, включая фракции, иммуноглобулины, в кровяной плазме для витаминов А, Е, С, каротина, глюкозы, мочевины, активностей энзимов (AST, GMT), минералий ( $\mathrm{Na}, \mathrm{K}, \mathrm{Ca}$, неорган. $\mathrm{P}, \mathrm{Zn}, \mathrm{Cw}, \mathrm{Fe})$. В коови телят были определены кислотно-основные величины крови $\left(\mathrm{pH}, \mathrm{pCO}_{2}, \mathrm{BE}, \mathrm{SB}\right)$, гематокрит, гемоглобин, число әритроцитов и лейкоцитов.

Гипогаммаглобулинемия, гиповитаминоз А и Е установлены у $50-75 \%$ телят. К важнейшим биохимическим показателям с диагностической точки зрения у телят принадлежит определение $\mathrm{Ig}$, витаминов, кислогно-щелочного равновесия крови, плазматического уровня глюкозы, мочевины, некоторых минеральных веществ и активности AST. 
Выбор клинико-биохимических показателей у телят должен быть целенаправленным. При исследовании и диагностике нарушения мегаболизма телят необходимо учитывать развитие иммунологических процессов, неустойчивость гомеостаза, функциональное несовершенство и незрелость органов и динамику зависимости от возраста теленка.

\section{References}

BOUDA, J.: Studium mechanismů acidobazických poruch u skotu a jejich význam $\mathrm{v}$ diagnostice. PhDr. Thesis, Brno, 1975, $185 \mathrm{p}$.

BOUDA, J.: Význam biochemických ukazatelủ pro diagnostiku onemocnèní telat a studium metabolických vztahủ mezi matkou a mládětem. Habilitation Thesis Brno, 1979, 143 p.

BOUDA, J. - JAGOŠ, P. - DVOŘÁK, V. - HAMŠfK, V.: Vitamin A and carotene in cows and their calves fed from buckets. Acta vet. Brno, 49, 1980: 45-52.

BOUDA, J. - DVOŘ́A, V. - MINKSOVÁ, E. - DVORÁK, R.: The activities of GOT, gamma-GT, alkaline phosphatase in blood plasma of cows and their calves. Acta vet. Brno, 49, 1980: 193-198.

JAGOŠ, P. - BOUDA, J. - ILLEK, J. - HOFIREK, B.: Rozpracování metabolických testů $\mathrm{k}$ určení skrytých poruch výměny látkové $u$ dojnic $\mathrm{v}$ podmínkách průmyslových komplexů. Project report, Brno 1975, $50 \mathrm{p}$.

JAGOŠ, P. - BOUDA, J. - DVOŘ́, V.: Vývoj zdravotního stavu telat $\mathrm{v}$ podmínkách velkovýrobních technologii. Project report, Brno, 1980, 63 p.

JAGOŠ, P. - BOUDA, J. - DVORÁK, V. - ILLEK, J. - HOFfREK, B.: Metody aktivní kontroly zdraví a včasné diagnostiky zdravotních poruch u telat. Veterinářství, 32, 1982: 250 to 252 .

KITCHENHAM, B. A. - ROWLANDS, G. J. - MANSTON, R. - DEW, S. M.: The blood composition of dairy calves reared under conventional and rapid-growth systems. Br. vet. J., 131, 1975: 436-446.

LAUERMAN, L. H. - RUPPANER, R. - NORMAN, B. B. - ADAMS, C. J.: Metabolic and cellular profile testing in calves under feedlot conditions. Protein fractions and lactate dehydrogenase isoenzymes - reference values. Am. J. vet. Res., 39, 1978: 855-857.

LUMSDEN, J. M. - MULLEN, K. - ROWE, R.: Hematology and biochemistry reference values for female Holstein cattle. Canad. J. comp. Med., 44, 1980: 24-31.

MAURER-SCHWEIZER, H. - WALSER, K.: Azidose und klinischer Zustand bei asphyktischen Kälbern. Berl. Münch. Tierärztl. Wschr., 90, 1977: 366-371.

McEWAN, A. D. - FISHER, E. W. - SELMAN, I. E. - PENHALE, W. J.: A turbidity test for estimation of immune globulin levels in neonatal calf serum. Clin. chim. Acta, 27, 1970: $155-163$.

McMURRAY, C. H. - LOGAN, E. F. - McPARLAND, P. J. - McRORY, F. J. - O'NEILL, D. G.: Sequential changes in some blood components in the normal neonatal calf. Br. J., 134, 1978: $590-597$.

RUPPANNER, R. - NORMAN, B. B. - ADAMS, C. J. - ADDIS, D. G. - LOFGREEN, G. P. - CLARK, J. G. - DUNBAR, J. R.: Metabolic and cellular profile testing in calves under feedlot conditions: minerals, electrolytes, and biochemical components - reference values. Am. J. vet. Res., 39, 1978: 841-844.

SLANINA, L. - BESEDA, I.: Metabolický profil hovädzieho dobytka vo vztahu $\mathrm{k}$ zdraviu a užitkovosti. Project report Košice, 1979.

VAGHER, J. P. - PEARSON, B. - BLATT, L. - KAYE, M.: Biochemical and hematologic values in male Holstein-Friesian calves. Am. J. vet. Res., 34, 1973: 273-277. 